\author{
Thomas C. Kwee \\ Taro Takahara \\ Reiji Ochiai \\ Rutger A. J. Nievelstein \\ Peter R. Luijten
}

\section{Diffusion-weighted whole-body imaging with background body signal suppression (DWIBS): features and potential applications in oncology}

Received: 6 December 2007

Revised: 24 January 2008

Accepted: 29 February 2008

Published online: 30 April 2008

(C) The Author(s) 2008

Electronic supplementary material The

online version of this article (doi:10.1007/

s00330-008-0968-z) contains supple-

mentary material, which is available

to authorized users.

T. C. Kwee $(\bowtie) \cdot$ T. Takahara .

R. A. J. Nievelstein · P. R. Luijten

Department of Radiology

(HP E01.132), University Medical

Center Utrecht,

Heidelberglaan 100,

3584 CX Utrecht, The Netherlands

e-mail: thomaskwee@gmail.com

Tel.: +31-30-2506688

Fax: +31-30-2581098

\section{R. Ochiai}

Department of Radiology,

Koga Hospital 21,

Kurume, Japan

\begin{abstract}
Diffusion-weighted magnetic resonance imaging (DWI) provides functional information and can be used for the detection and characterization of pathologic processes, including malignant tumors. The recently introduced concept of "diffusion-weighted whole-body imaging with background body signal suppression" (DWIBS) now allows
\end{abstract}

acquisition of volumetric diffusionweighted images of the entire body. This new concept has unique features different from conventional DWI and may play an important role in wholebody oncological imaging. This review describes and illustrates the basics of DWI, the features of DWIBS, and its potential applications in oncology.

Keywords Diffusion magnetic resonance imaging .

Whole-body imaging · Oncology

Neoplasm staging

\section{Introduction}

Cancer is the second leading cause of death in developed countries, is among the three leading causes of death for adults in developing countries, and is responsible for $12.5 \%$ of all deaths worldwide [1]. Once a malignant tumor is detected, determination of disease extent (staging) is important for appropriate treatment planning and determining prognosis. Imaging plays a pivotal role in cancer staging. Furthermore, imaging is of great importance in monitoring response to therapy and in the detection of tumor recurrence.

Diffusion-weighted magnetic resonance imaging (DWI) provides functional information and can be used for the detection and characterization of pathologic processes, including malignant tumors; it may therefore be of value in staging and follow-up imaging of malignant tumors. DWI using single-shot echo-planar imaging (EPI) is a wellestablished method to examine the brain. Extracranial DWI, however, did not become a clinical standard because the use of EPI was complicated by magnetic susceptibility artifacts and severe image distortion in the body [2-6]. Recently introduced parallel imaging techniques, such as sensitivity encoding (SENSE) $[7,8]$, and the development of stronger gradients and multichannel coils have largely overcome this problem; DWI of adequate quality can now be performed in the body, at high $b$-value $[9,10]$. Despite the above-mentioned breakthroughs in DWI, breathhold or respiratory triggered scanning was still considered necessary since it was widely accepted that respiratory motion was an impediment for DWI of (moving) visceral organs [11-15].

Then, in 2004, Takahara et al. [16] reported a unique concept of whole-body DWI, called "diffusion-weighted whole-body imaging with background body signal suppression" (DWIBS). This technique intentionally uses free breathing scanning rather than breathholding or respiratory triggering to visualize (moving) visceral organs and their lesions. In a later published article, Ballon et al. [17] also 
reported whole-body DWI during free breathing. The study by Ballon et al. [17] aimed to visualize metastatic lesions in static tissue (bone marrow); this was in agreement with accepted theory at that time. However, they additionally found that visceral organs, such as the spleen and kidneys, were also visualized [17]. The feasibility of the free breathing approach could initially not be explained, but was clarified later; it can be understood with knowledge of the relationship between the motion probing gradients (MPGs) and the type (coherence) of motion, as will be described in a later section. This concept offers a wide range of potential applications in whole-body oncological imaging.

The first purpose of this review is to acquaint the reader with the features of DWIBS. Second, although evidence regarding its effectiveness is still limited, the potential applications of DWIBS in oncology will be described and illustrated. Finally, drawbacks, limitations, and future considerations of DWIBS will be discussed.

\section{Basics of DWI}

As introduced by Stejskal and Tanner [18], the most common approach to render magnetic resonance imaging (MRI) sensitive to diffusion is to place two strong symmetrical gradient lobes (so-called MPGs) on either side of the $180^{\circ}$ refocusing pulse in a spin-echo sequence (Figs. 1, 2, 3). The degree of signal attenuation in DWI depends both on the magnitude of diffusion (also referred to as intravoxel "incoherent" motion [19-21]) and the amount of diffusion weighting. It should be realized that

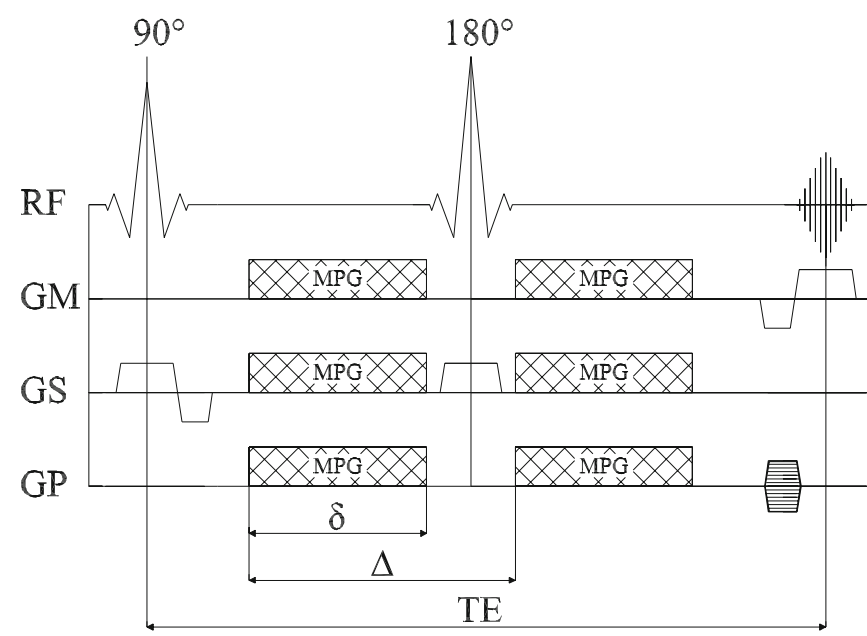

Fig. 1 The Stejskal-Tanner sequence [5] ( $R F$ radio frequency, $G M$ readout direction, $G P$ phase encoding direction, $G S$ section select direction, $M P G$ motion probing gradient, $\delta$ duration of one MPG, $\Delta$ time interval between the leading edges of the MPGs, $T E$ echo time) only intravoxel "incoherent" motion leads to signal decrease, whereas intravoxel "coherent" motion, irrespective of its magnitude, does not affect signal, as will be explained in a later section. The amount of diffusion weighting is determined by the so-called $b$-value $(b)$ and is calculated as follows:

$b=\gamma^{2} G^{2} \delta^{2}(\Delta-\delta / 3)$,

where $\delta$ is the duration of one MPG, $\Delta$ is the interval between the leading edges of the MPGs, $G$ is the strength of the MPG (Fig. 1), and $\gamma$ is the gyromagnetic ratio (the ratio of the magnetic moment to the angular momentum of a particle, $42.58 \mathrm{MHz} / \mathrm{T}$ for hydrogen) [7, 18].

In biological tissue, diffusion is expressed and quantified by means of an effective or apparent diffusion coefficient (ADC). If two or more images with different $b$-values are acquired, the ADC of a selected region-of-interest (ROI) can be calculated as follows:

$A D C=\left(1 / b_{1}-b_{0}\right) \ln \left(S\left[b_{1}\right] / S\left[b_{0}\right]\right)$,

where $b_{1}$ and $b_{0}$ represent two different $b$-values, $S\left[b_{1}\right]$ the signal intensity of the selected ROI on the slice level acquired with $b$-value $b_{1}$, and $S\left[b_{0}\right]$ the signal intensity of the same ROI on the same slice level acquired with $b$-value $b_{0}$. A pixel by pixel map (so-called ADC map), whose intensity yields quantitative estimation of the regional $\mathrm{ADC}$, can be obtained by post-processing [22].

DWI has potential in the field of oncological imaging. Malignant tumors may exhibit either restricted or increased diffusion, depending on their micro-architecture. For example, tumors with a high cellular density possess more cellular membranes per volume unit, which impedes the mobility of water molecules and restricts diffusion. Failure of the $\mathrm{Na}^{+} / \mathrm{K}^{+}$ATPase pump during necrotic cell death (similar to acute ischemic stroke) may also lead to restricted diffusion. Restricted diffusion has been reported for most malignant tumors $[9,10,23]$. On the other hand, loss of cell membrane integrity in necrotic tumors and decrease in tumor cellularity due to necrosis and/or apoptotic processes may result in increased diffusion, unless the necrotic fluid itself possesses high viscosity. In addition, intratumoral edema and cystic tumor components also increase diffusion because of increased water content $[9,10,23]$.

For a more in-depth overview of the current status of conventional (breathhold or respiratory triggered) DWI, we refer the reader to the articles by Thoeny and De Keyzer [9], and Koh and Collins [10].

\section{Concept of DWIBS}

When DWI was applied to the chest and abdominal region, another important problem was encountered: respiratory 


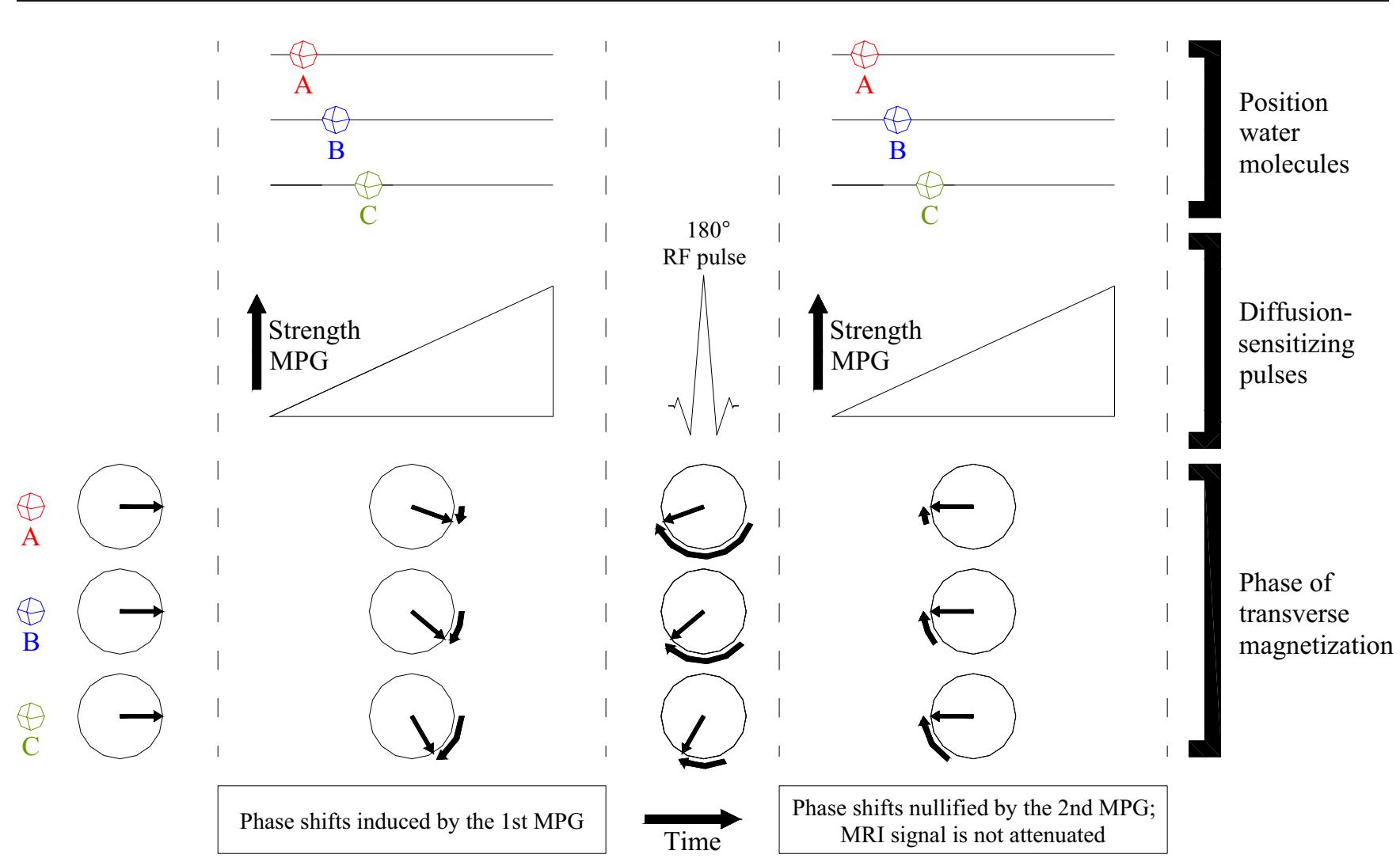

Fig. 2 Three stationary water molecules $(A, B, C)$ in one voxel. Phase shifts induced by the first motion probing gradient $(M P G)$ are nullified by the second MPG. Because of the maintained phase coherence among the three water molecules, the MRI signal is not attenuated ( $R F$ radio frequency) motion. It was known that T2-weighted image contrast could be maintained in T2-weighted imaging during free breathing. Nevertheless, breathholding or respiratory triggering was preferred to avoid image blurring. Similar rules seem to apply to DWI, but with a different underlying theory. Most people believed that diffusion-weighted image contrast would largely be lost in DWI during free breathing, because respiratory motion is tremendously larger than diffusion. Thus, DWI during free breathing seemed to be impossible and breathholding or respiratory triggering was strongly recommended, mainly to avoid signal loss rather than image blurring. Because of its limited scanning time, only a limited number of slices with relatively large slice thickness could be obtained in each examination, similar to incremental computed tomography (CT). As a result, the signal-to-noise ratio (SNR) is low and lesion conspicuity may be diminished. Respiratory triggered scanning improves SNR, but at the expense of an approximately threefold increase in scanning time.

As described previously, Takahara et al. [16] developed the concept of DWIBS and proved the feasibility of DWI during free breathing. They also observed that SNR of DWI during free breathing considerably surpassed that of breathhold scanning [16]. These observations were in contradiction with the general assumption at that time. They extended the possibilities of DWI: scanning time is no more limited (as in breathhold scanning) and image acquisition time is no more confined to a particular phase of the breathing cycle (as in respiratory triggered scanning), images with multiple $b$-values including high $b$-values around $1,000 \mathrm{~s} / \mathrm{mm}^{2}$ can be acquired, thin slices can be obtained, and multiple signal averaging is possible. Furthermore, these advances enable volumetric [three-dimensional (3D)] image processing, including maximum intensity projections (MIPs), volume rendering, and multiplanar reformatting (MPR) in any plane (Figs. 4 and 5).

\section{Reason for the feasibility of free breathing in DWI}

Sensitivity of DWI to microscopic diffusion (in the order of several micrometers) is maintained even during free breathing. To understand the feasibility of free breathing in DWIBS, two types of motion should be distinguished: intravoxel "incoherent" motion and intravoxel "coherent" motion. Intravoxel "incoherent" motion refers to the 


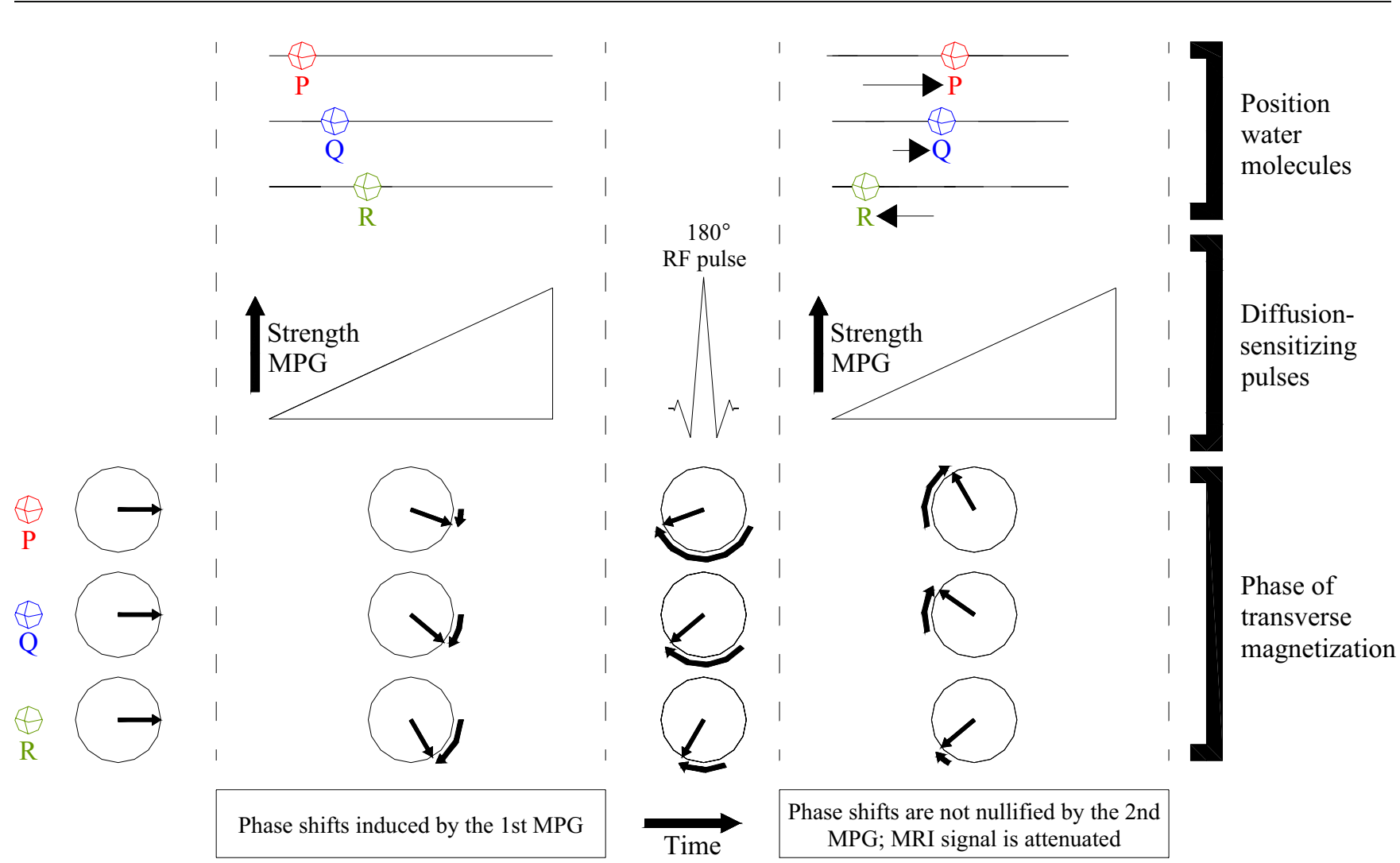

Fig. 3 Three water molecules $(P, Q, R)$ in one voxel, undergoing diffusion. Phase shifts induced by the first motion probing gradient $(M P G)$ are not nullified by the second MPG. Because of the resulting phase dispersion among the three water molecules, the MRI signal is attenuated ( $R F$ radio frequency) incoherent motion of water molecules within a voxel (i.e., diffusion) [19-21]. Intravoxel "coherent" motion refers to the coherent motion of water molecules within a voxel. Since the two MPGs in a diffusion-weighted sequence (Fig. 1) are applied within a very small time interval (in the order of $50 \mathrm{~ms}$ ), additional respiratory motion can indeed be regarded as coherent motion. The two MPGs only lead to signal decrease in case of intravoxel "incoherent" motion (i.e. diffusion), whereas signal is not affected by intravoxel "coherent" motion caused by additional respiratory motion (Movie clip 1). In other words, because DWIBS employs single-shot EPI, the acquired phase shift due to respiratory motion is equal in each phase-encoding step and, therefore, does not affect image formation (Fig. 6) [7]. Thus, DWI can be regarded as equal to T2-weighted imaging with respect to respiratory motion. In other words, diffusionweighted image contrast is maintained during free breathing, while breathholding or respiratory triggering reduces image blurring. However, the SNR in DWI is significantly less than in T2-weighted imaging. Therefore, instead of aiming to reduce image blurring, it can be more advantageous to improve the SNR in DWI by means of free breathing scanning, which allows thin slice acquisitions and multiple-slice excitations for volumetric imaging.

\section{Practical implementation of DWIBS}

Suggested sequences for DWIBS at $1.5 \mathrm{~T}$ are listed in Table 1. It is currently recommended to obtain axial slices in DWIBS, to minimize image distortion. Because DWIBS allows obtaining thin axial slices (up to $4 \mathrm{~mm}$ ), high quality reformatted images can subsequently be created in any plane. DWIBS is combined with either a short inversion time inversion recovery (STIR) pre-pulse or a frequencyselective [chemical shift selective (CHESS)] pre-pulse for fat suppression. STIR theoretically gives superior global fat suppression over an extended field of view, compared with CHESS, because of its insensitivity to magnetic field inhomogeneities [24]. Therefore, STIR can be used everywhere in the body. In addition, STIR may be useful in suppressing bowel signal, which may have a short T1 value, similar to that of fat. However, one of the main disadvantages of STIR, compared with CHESS, is decreased SNR because of partial loss of proton signal during the inversion time [24]. Since abdominal organs usually do not suffer significantly from magnetic field inhomogeneities, CHESS can be used in this area, both to increase SNR and to reduce image acquisition time. Continuous whole-body imaging, however, usually re- 


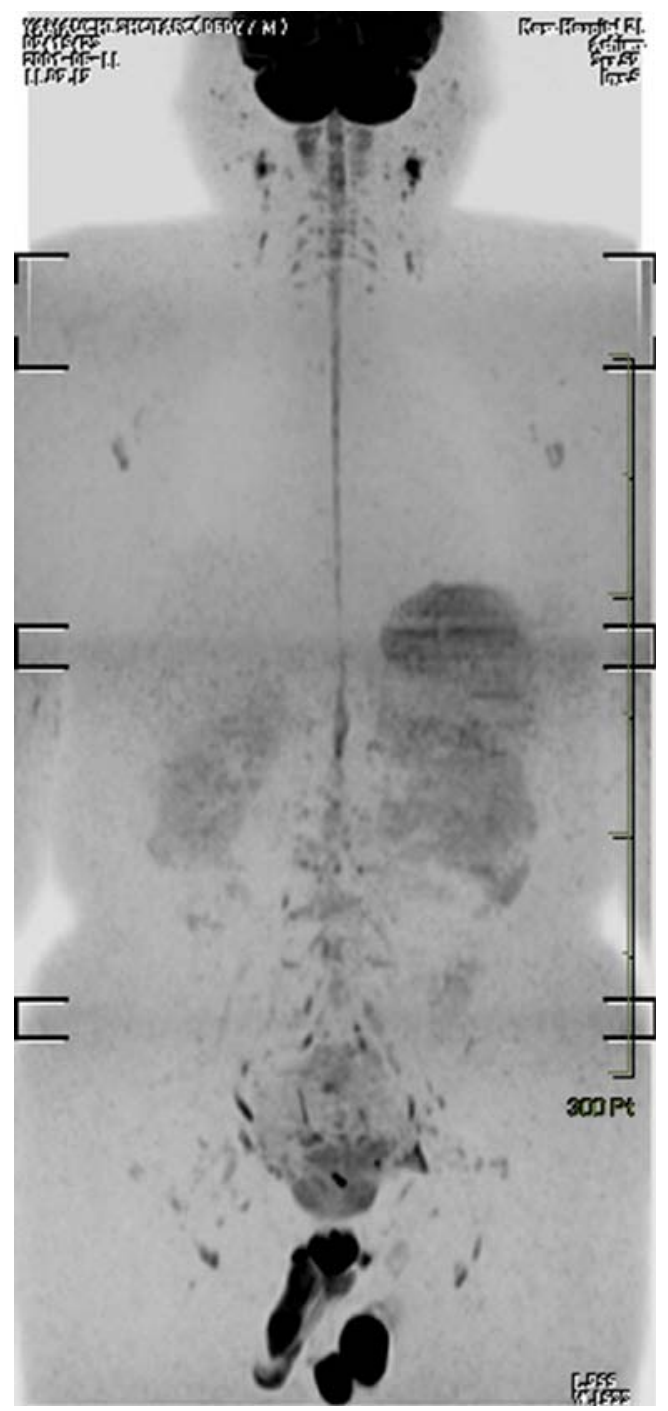

Fig. 4 Coronal maximum intensity projection DWIBS image (inverted black-and-white gray scale) of a healthy 60-year-old male volunteer. Normal high signal intensity (black on this image) can be seen in the brain, spinal cord, nerves of the brachial plexus, prostate, testis, penis, spleen, and cervical, axillary, pelvic, and inguinal lymph nodes

quires the use of STIR because of the presence of magnetic field inhomogeneities in the neck, shoulder, lungs, and lower extremities. On the other hand, it is possible to optimize a whole-body examination by imaging each station with the most appropriate fat suppression technique (either STIR or CHESS). The $b$-value determines the degree of background body signal suppression; $b$-values in the range of $800-1,000 \mathrm{~s} / \mathrm{mm}^{2}$ generally result in good background body signal suppression for tumor detection. It should be realized that the use of a certain $b$-value combined with STIR is more effective in background body signal suppression than a combination of the same $b$-value with CHESS. For homogeneous organs like the liver and pancreas, background body signal suppression is of less importance, and a $b$-value of $500 \mathrm{~s} / \mathrm{mm}^{2}$ is sufficient for tumor detection in the majority of cases, while reducing image acquisition time. On the other hand, for tumor detection in organs with an already (normal) high signal intensity in DWIBS, such as the prostate, $b$-values in the order of $1,500-2,000 \mathrm{~s} / \mathrm{mm}^{2}$ can be applied. For signal reception in DWIBS, either a built-in body coil or a surface coil can be used. Advantages of a built-in body coil are patient comfort and large coverage. However, although it has been reported that image quality is clear enough for the detection of lesions [25], the contrast-to-noise ratio (CNR) is inferior to that of a surface coil. One approach to obtain whole-body images using a surface coil is the use of a socalled whole-body surface coil design. A whole-body surface coil design combines a large number of seamlessly integrated coil elements and independent radiofrequency channels, which offers large anatomical coverage (in the order of $200 \mathrm{~cm}$ ) and allows image acquisition without any time loss due to patient or coil repositioning. However, is it also possible to perform a time-efficient whole-body examination using only one surface coil with limited coverage. In order to perform such an examination, an additional table platform should be mounted on top of the original patient table. Furthermore, spacers should be placed between both tables to create space to move the lower part of the surface coil from one station to the next. As a result, the patient can remain in the same position, repositioning of the surface coil to image the next station requires only little additional time ( $<1 \mathrm{~min}$ per station), and $3 \mathrm{D}$ alignment among the imaged stations is maintained. After completion of the examination, separately imaged stations can be merged with sophisticated software, creating the whole-body image (Fig. 7). Image acquisition time for DWIBS is typically in the order of 5-7 min per 25$\mathrm{cm}$ coverage, when using STIR and a $b$-value of $1,000 \mathrm{~s} /$ $\mathrm{mm}^{2}$. Inverting the gray scale of DWIBS images makes them resemble PET-like images (Figs. 4, 5). DWIBS can be performed on state-of-the-art MRI systems supplied by all major vendors, including GE Healthcare, Hitachi Medical Systems, Philips Medical Systems, Siemens Medical Solutions, and Toshiba Medical Solutions.

\section{Potential applications of DWIBS in oncology}

\section{Staging}

Primary tumors and distant metastases exhibiting restricted diffusion can be identified with DWIBS. DWIBS may especially be useful to detect relatively small lesions because of its high CNR. It should be noted, however, that evaluation of areas close to the heart and the diaphragm may be hampered in DWIBS because of signal loss and artifacts due to incoherent tissue motion. Nevertheless, overall, DWIBS may be a very useful adjunct to anatomical 
Table 1 Suggested sequences for DWIBS at $1.5 \mathrm{~T}(++$ very good, + good, $+/-$ moderate, - poor, -- very poor; $F O V$ field of view, $R F O V$ rectangular FOV, $N A$ not applicable, $T E$ echo time, $T I$ inversion time, $T R$ repetition time)

\begin{tabular}{|c|c|c|}
\hline Name & DWIBS-STIR & DWIBS-CHESS \\
\hline Main characteristics & $\begin{array}{l}\text { Most suitable for organs and body regions } \\
\text { suffering from large magnetic field } \\
\text { inhomogeneities } \\
\text { Useful in suppressing bowel signal } \\
\text { Relatively lower SNR than DWIBS-CHESS } \\
\text { Relatively more time-consuming than DWIBS-CHESS }\end{array}$ & $\begin{array}{l}\text { Most suitable for organs and body regions } \\
\text { without significant magnetic field } \\
\text { inhomogeneities } \\
\text { Relatively higher SNR than DWIBS-STIR } \\
\text { Relatively less time-consuming than DWIBS-STIR }\end{array}$ \\
\hline \multicolumn{3}{|l|}{ Suitability } \\
\hline Neck and shoulder & ++ & - \\
\hline Chest & ++ & + \\
\hline \multicolumn{3}{|l|}{ Abdomen and pelvis } \\
\hline Liver, pancreas, spleen & + & ++ \\
\hline Colon & ++ & + \\
\hline Kidneys & ++ & + \\
\hline Uterus, bladder & + & ++ \\
\hline Prostate & + & + \\
\hline Extremities & ++ & + \\
\hline \multicolumn{3}{|l|}{ Imaging parameters } \\
\hline Fat suppression & STIR & CHESS \\
\hline$b$-value $\left(\mathrm{s} / \mathrm{mm}^{2}\right)$ & $1,000^{\mathrm{a}, \mathrm{b}}$ & $1,000^{\mathrm{a}, \mathrm{b}}$ \\
\hline Direction of MPGs & Phase, frequency, and slice & Phase, frequency, and slice \\
\hline TR (ms) & 10,205 & 7,800 \\
\hline TE (ms) & 70 & 70 \\
\hline $\mathrm{TI}(\mathrm{ms})$ & 180 & NA \\
\hline Parallel imaging factor & 2 & 2 \\
\hline Halfscan factor & 0.6 & 0.6 \\
\hline EPI factor & 47 & 47 \\
\hline Slice orientation & Axial & Axial \\
\hline No. of slices & 60 & 60 \\
\hline Slice thickness/gap (mm) & $4 / 0$ & $4 / 0$ \\
\hline FOV (mm) & 400 & 400 \\
\hline RFOV (\%) & 70 & 70 \\
\hline Matrix & 160 & 160 \\
\hline Scan percentage & $70 \%$ & $70 \%$ \\
\hline Actual voxel size $\left(\mathrm{mm}^{3}\right)$ & $2.5 \times 3.6 \times 4.0$ & $2.5 \times 3.6 \times 4.0$ \\
\hline Calculated voxel size $\left(\mathrm{mm}^{3}\right)$ & $1.6 \times 1.6 \times 4.0$ & $1.6 \times 1.6 \times 4.0$ \\
\hline No. of signals averaged & 10 & 6 \\
\hline Scan time & $7 \min 8 s$ & $3 \min 2 \mathrm{~s}$ \\
\hline
\end{tabular}

${ }^{\text {a}}$ For imaging of the upper abdomen (including liver, spleen, and pancreas), a $b$-value of $500 \mathrm{~s} / \mathrm{mm}^{2}$ can be applied, if necessary combined with respiratory triggered scanning

${ }^{\mathrm{b}}$ For tumor detection in the prostate, $b$-values in the order of $1,500-2,000 \mathrm{~s} / \mathrm{mm}^{2}$ can be applied

MRI or CT, where small lesions may be obscured or overlooked because of the large amount of image data, and lesions in normal-sized organs may be missed (Fig. 8).

Accurate assessment of nodal status has important therapeutic and prognostic significance in patients with newly diagnosed cancers. Current cross-sectional imaging modalities rely on insensitive size and morphologic criteria and, thus, lack the desired accuracy for characterizing lymph nodes [26]. DWIBS may be a valuable tool for the identification and characterization of lymph nodes; normal lymph nodes have a relatively restricted diffusion because of their high cellular density and are well visualized with 


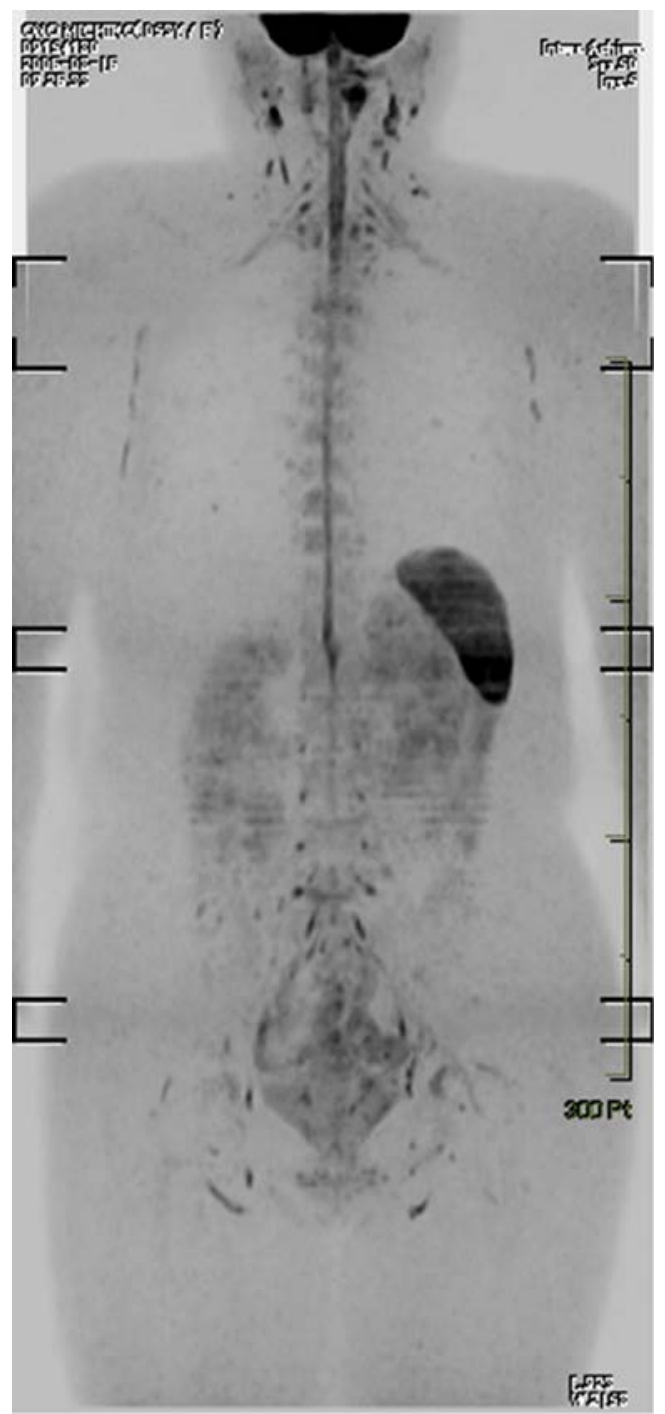

Fig. 5 Coronal maximum intensity projection DWIBS image (inverted black-and-white gray scale) of a healthy 53-year-old female volunteer. Normal high signal intensity (black on this image) can be seen in the brain, spinal cord, nerves of the brachial and lumbosacral plexus, vertebral bone marrow, endometrium, spleen, and cervical, axillary, pelvic, and inguinal lymph nodes

DWIBS, while surrounding fat tissue is suppressed (Figs. 4, 5). Metastatic lymph nodes may have increased cellular density or necrotic areas, which further restricts or increases diffusion, respectively. However, it is still unknown whether it is possible to differentiate healthy lymph nodes or benign lymphadenopathy from malignant lymph nodes using DWIBS with calculation of ADCs. Nevertheless, DWIBS is an outstanding tool to identify lymph nodes, irrespective of their histological composition. DWIBS, for example, may be complimentary to nanoparticle-enhanced MRI, which has proven to be very useful for the characterization of lymph nodes [27] but may have difficulties in tracing them.

\section{Monitoring response to therapy}

Another potential application of DWIBS is in the assessment of radiation and/or chemotherapy efficacy. Currently, CT and anatomical MRI are the most commonly used means for the follow-up of cancer patients. Serial measurements of tumor dimensions are made, and criteria, such as the World Health Organization (WHO) or Response Evaluation Criteria in Solid Tumors (RECIST) criteria are applied to assess therapeutic efficacy [28]. DWIBS can similarly be applied and its ability to obtain (automated) total volume measurements may especially be useful (Fig. 9). In the near future, it is expected that the further development of multichannel coils and accelerated parallel imaging will allow acquisition of true isotropic high-resolution volume data sets.

A disadvantage of the current approach of obtaining repetitive tumor size measurements on CT or MRI is that it cannot be used to give an early assessment of the effectiveness of radiation and/or chemotherapy. Early identification of patients who are likely to show a poor response to a certain treatment can be of great advantage: it provides the opportunity to adjust individual treatment regimes more rapidly, and sparing patients unnecessary morbidity, expense and delay in initiation of effective treatment. PET is a promising functional imaging modality for the early assessment of response to therapy for various tumors [29], but DWIBS, which is less expensive and does not require any patient preparation, may also be of value. Similar to PET, DWIBS is able to detect functional changes in areas involved with tumor before structural changes become visible (Fig. 10). In addition, the recently introduced functional diffusion map (fDM) allows calculation and visualization of tumor ADC changes during treatment and takes into account spatial heterogeneity of tumor response; it has proved to be useful to predict treatment response in brain tumor patients [30-32] and its feasibility for evaluating treatment response of metastatic bone disease from prostate cancer has recently been presented [33]. Extracranial application of fDM analysis in a DWIBS examination would be very attractive, but is technologically challenging and requires further investigation. The accuracy and reproducibility of ADC mapping and measurements in DWIBS, which are the primary requisites for fDM analysis, should first be investigated.

\section{Detection of tumor persistence or recurrence}

Differentiating persistent or recurrent tumor tissue from nontumoral posttherapeutic change is of great importance for correct patient management, but is often difficult with CT or anatomical MRI. Functional imaging techniques, such as PET [34] may allow better differentiation. The less expensive DWIBS may also be of value. It can be hypothesized that persistent or recurrent tumor tissue will 


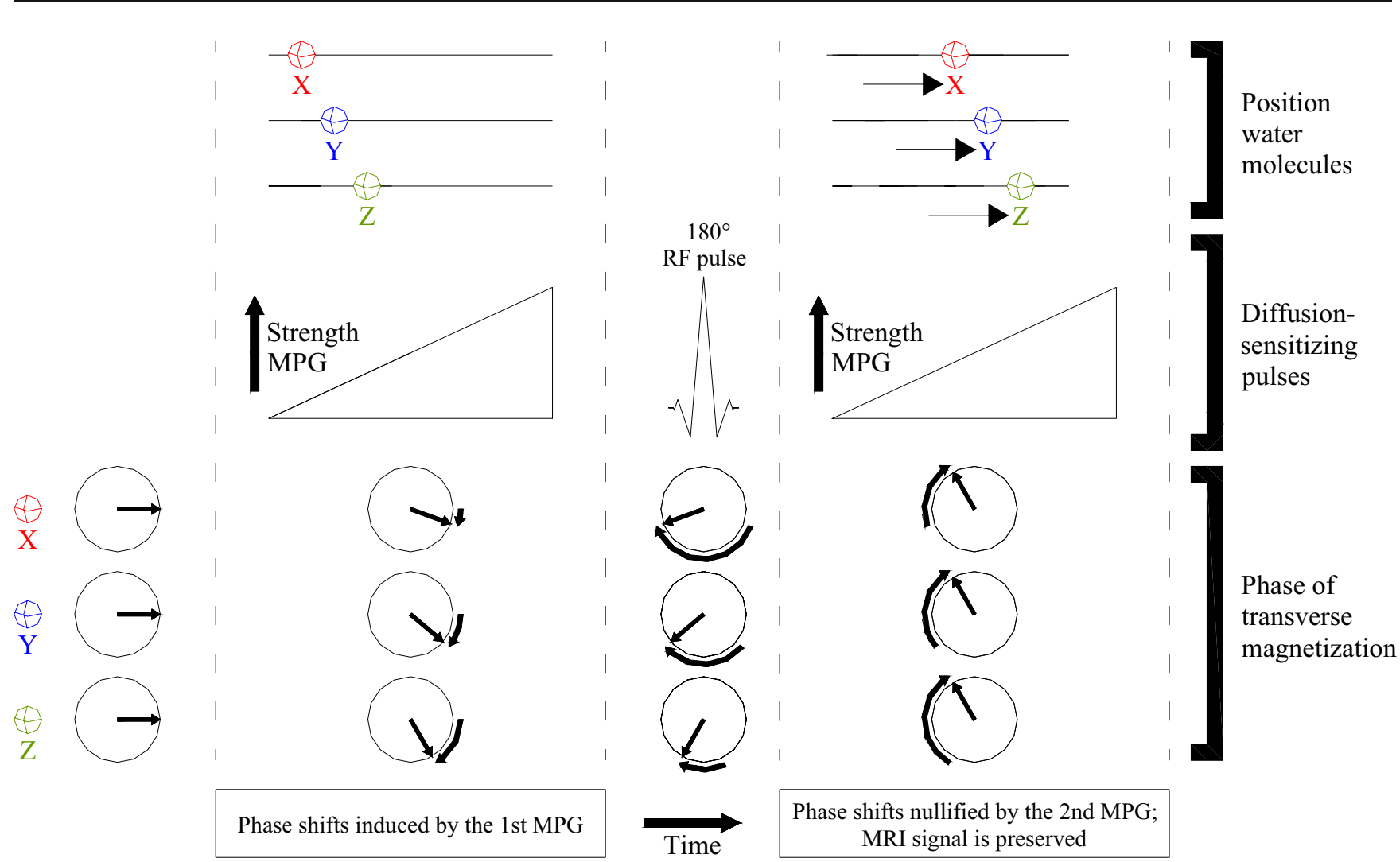

Fig. 6 Three stationary water molecules $(X, Y, Z)$ in one voxel, undergoing respiratory motion. Respiratory motion induces a linear phase shift but does not cause phase dispersion. Because of the maintained phase coherence among the three water molecules, MRI signal is preserved ( $M P G$ motion probing gradient, $R F$ radio frequency)

Ichikawa et al. [36] also investigated the value of DWIBS in the detection of pancreatic adenocarcinoma. Twenty-six patients with pathologically proven pancreatic adenocarcinoma and another 23 controls with either chronic pancreatitis $(n=20)$ or intraductal papillary mucinous tumors $(n=3)$, without any evidence of pancreatic adenocarcinoma during a minimum of 12 months of clinicoradiological follow-up, underwent DWIBS. Visual assessment of the DWIBS images resulted in high sensitivity $(96.2 \%, 75 / 78)$ and specificity $(98.6 \%, 68 / 69)$.

Tsushima et al. [37] investigated the value of DWIBS, T2-weighted MRI, and DWIBS/T2-weighted MRI fusion as a screening tool for the detection of abdominal malignancies. Thirty-seven patients with a malignant tumor (five renal cell carcinomas, four advanced gastric carcinomas, four metastatic liver tumors, three pancreatic carcinomas, two hepatocellular carcinomas, two cholangiocarcinomas, one bone lesion of lymphoma, one transverse colon carcinoma, five rectal carcinomas, three ovarian carcinomas, two prostate carcinomas, two metastatic bone tumors, one peritoneal dissemination, one recurrent colon carcinoma, and one ascending colon carcinoma) and 73 patients without a malignant tumor were retrospectively analyzed. Malignant tumors had ever, because no benign pathologies were included the specificity of DWIBS might have been overestimated [35]. 
Fig. 7 Separately imaged stations can be merged with sophisticated software, creating the whole-body image

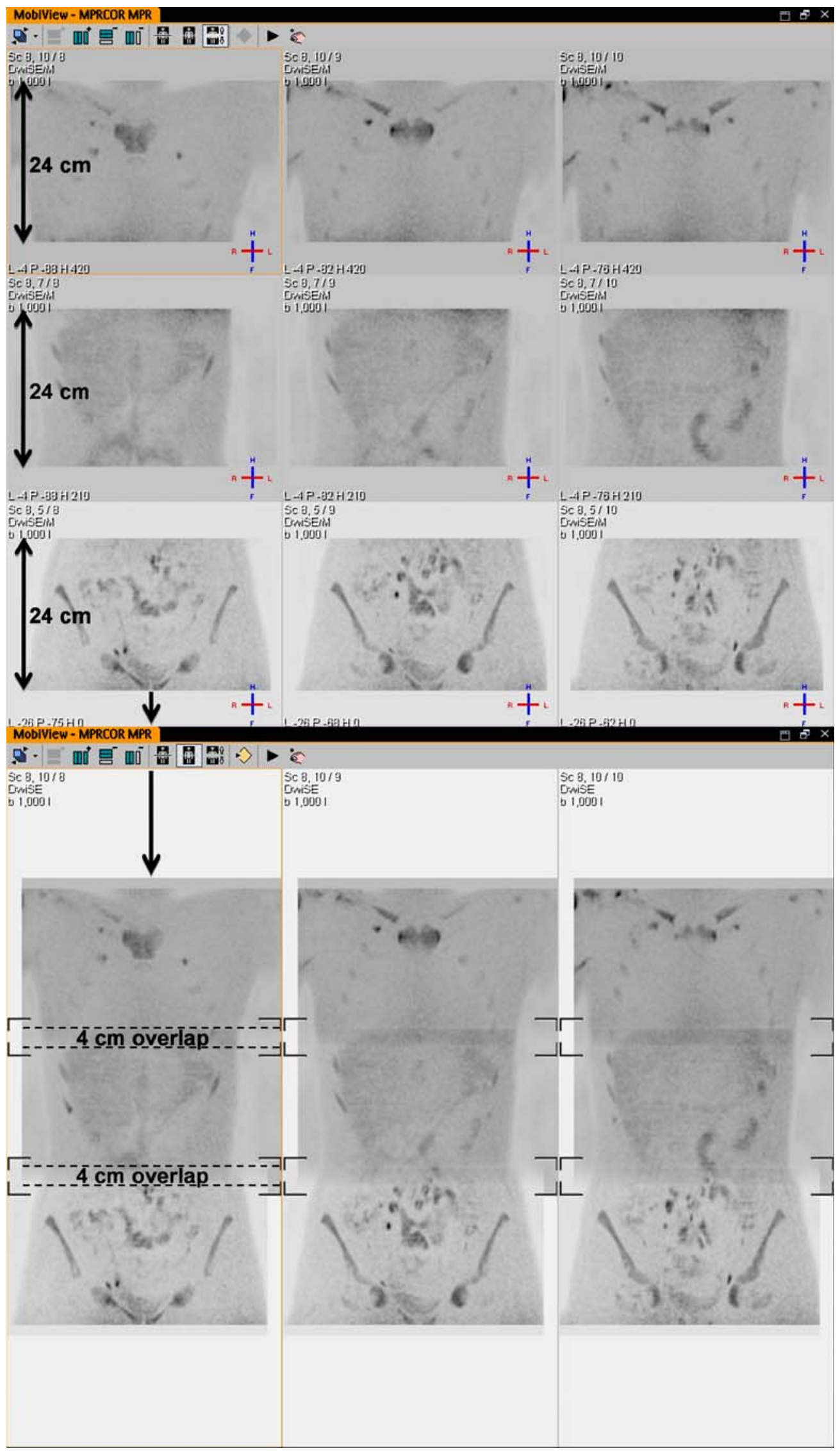




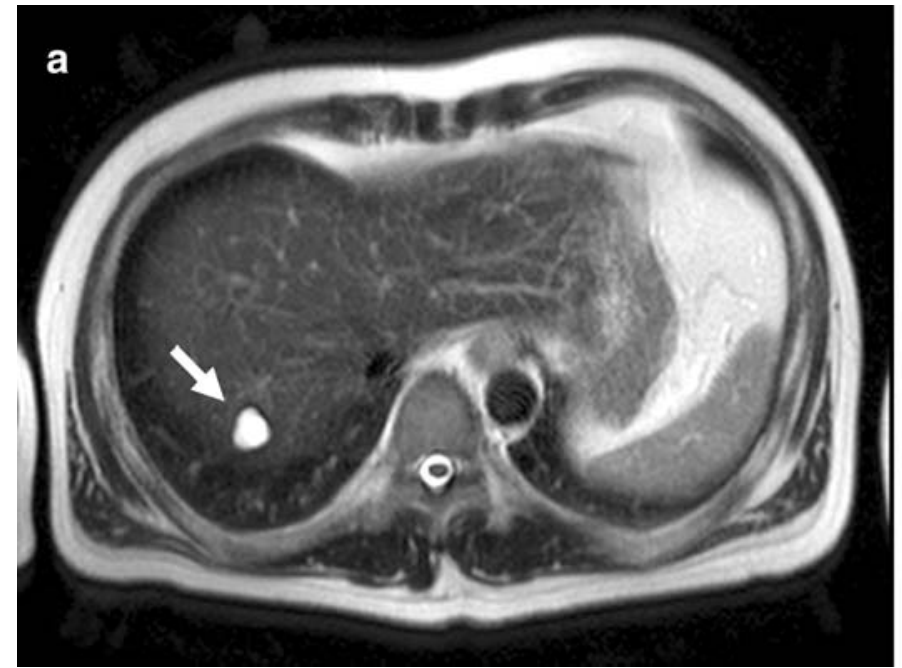

Fig. 8 A 41-year-old man with distal esophageal cancer shown by DWIBS but inconspicuous on T2-weighted MRI. a Axial T2weighted image shows a small high signal intensity lesion in segment 7 of the liver (arrow), consistent with a cyst. No other b

conspicuous lesions can be identified. b Axial DWIBS image (inverted black-and-white gray scale) at the same level shows abnormal signal intensity in the lower part of the esophagus (arrow), consistent with esophageal carcinoma

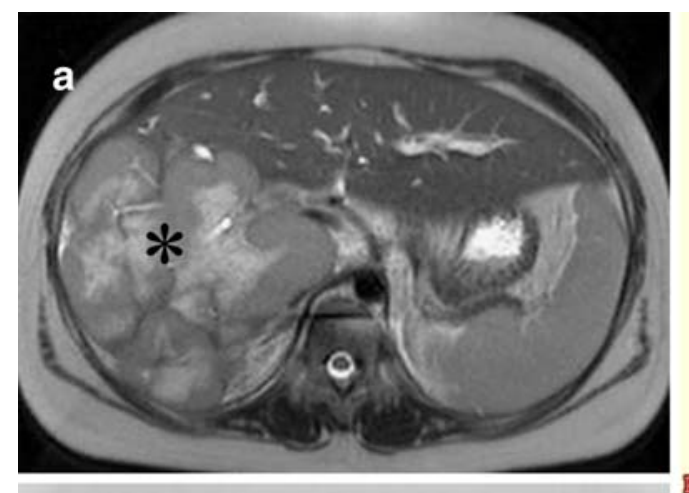

C

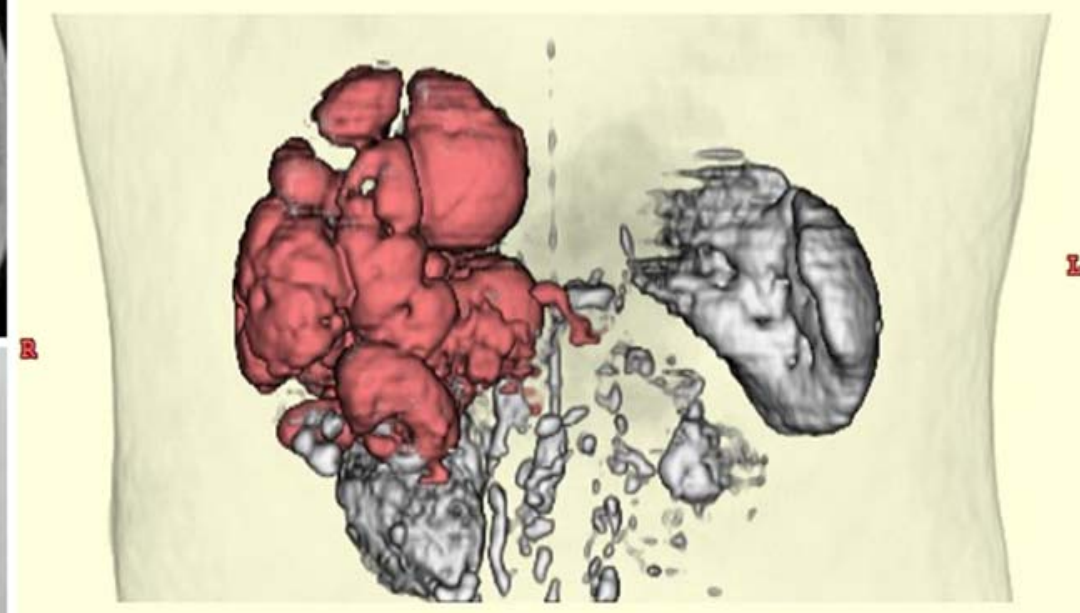

\&
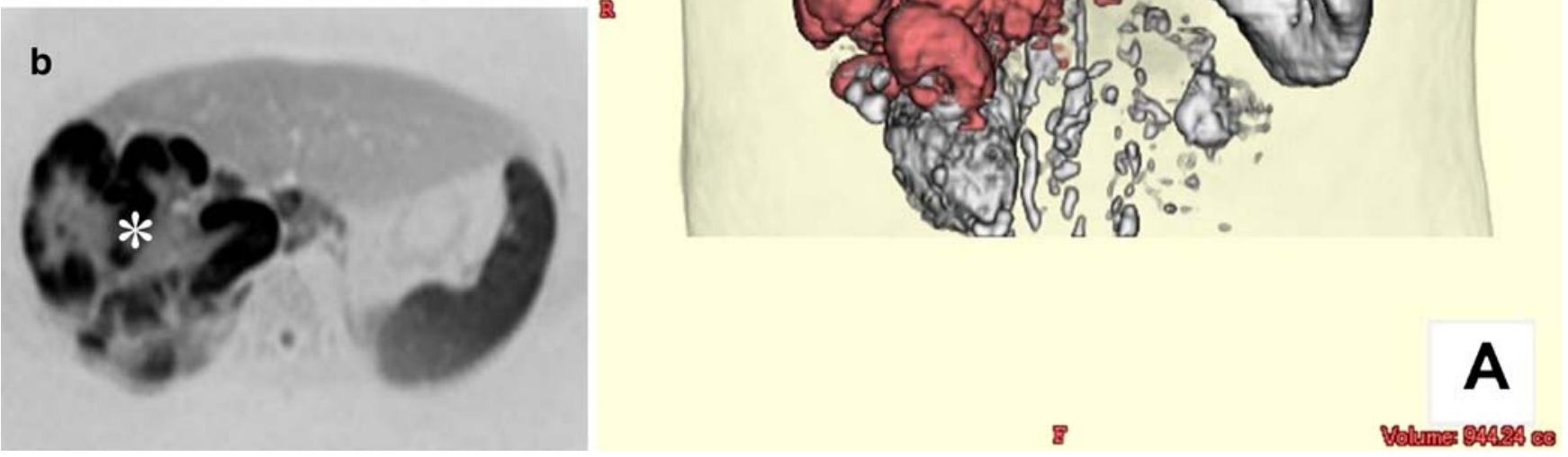

Fig. 9 Volume measurement of a primary hepatic diffuse large Bcell lymphoma in a 10-year-old girl using DWIBS. a Axial respiratory-gated T2-weighted image shows a bulky mass occupying most of the right lobe of the liver (asterisk). b Axial DWIBS image (inverted black-and-white gray scale) at the same level clearly

visualizes the tumor with excellent contrast between the tumor and surrounding tissue (asterisk). c Volume-rendered image using thin slice (4 mm, no gap) DWIBS dataset shows the three-dimensional shape of the tumor (colored red) and allows for tumor volume measurement 

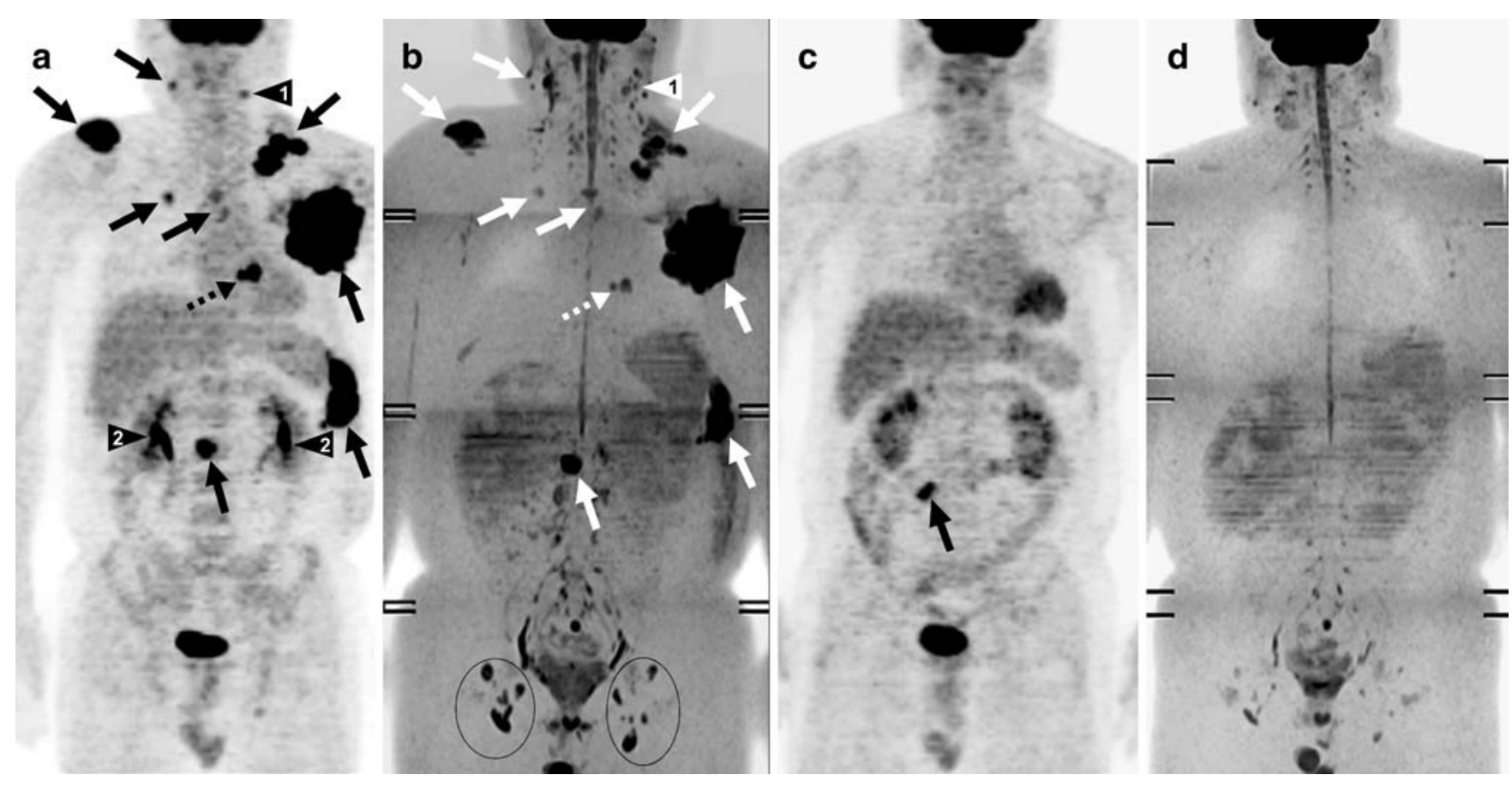

Fig. 10 Comparison of pre- and post-chemotherapy FDG-PET and DWIBS images in a 44-year-old man with diffuse large B-cell lymphoma. a Coronal maximum intensity projection FDG-PET and b coronal maximum intensity projection DWIBS images before initiation of chemotherapy. Both images show cervical, bilateral supra/infraclavicular, mediastinal, left axillary, para-aortic lymph node, and splenic involvement (arrows), and cardiac involvement (dashed arrow). c FDG-PET and d DWIBS images at the end of treatment show resolvement of all pre-existing lesions. a, b A limitation of DWIBS is that discrimination between normal and metastatic lymph nodes is still based on size criteria; the FDG-PET positive left cervical lymph node (arrowhead 1) cannot conclu-

pathologically been verified and the absence of a malignancy in the controls was confirmed by clinical and radiological (conventional MRI and contrast-enhanced CT) findings. The areas under the receiver-operating characteristic curves for the visual detection of malignant lesions of DWIBS/T2-weighted MRI fusion (0.904) was significantly higher than those of DWIBS $(0.720, P<0.01)$ and T2weighted MRI $(0.822, P<0.05)$. Both sensitivity and specificity were higher in DWIBS/T2-weighted MRI fusion $(89.5 \%$ and $81.9 \%$, respectively) compared with those of DWIBS $(72.4 \%$ and $59.0 \% ; P<0.01$ and $P<0.001$, respectively), and the specificity of DWIBS/T2-weighted MRI fusion $(81.9 \%)$ was significantly higher than that of T2-weighted MRI $(68.8 \%, P<0.01)$. Limitations of this study are its retrospective design, the presence of selection bias, the use of an imperfect reference standard, and the heterogeneity of the patient population [37].

Komori et al. [38] compared the lesion detection rates of DWIBS and ${ }^{18} \mathrm{~F}$-fluoro-2-deoxyglucose (FDG)-PET/CT in 16 consecutive patients with a total of 27 malignant lesions (15 lung cancers, five pulmonary metastases of parathyroid cancer, three pulmonary metastases of lung cancer, three

sively be identified as malignant on DWIBS (arrowhead 1). DWIBS also shows prominent bilateral inguinal lymph nodes (encircled) which are normal according to FDG-PET. On the other hand, thanks to its higher spatial resolution, DWIBS visualizes two separate cardiac lesions (dashed arrow), whereas FDG-PET shows only one large cardiac lesion (dashed arrow). DWIBS also allows better evaluation of the urinary tract than FDG-PET, where potential lesions can be obscured because of FDG accumulation (arrowheads 2). c Note the physiological FDG uptake in the large intestine (arrow), which should not be confused with persistent malignant lymphoma

colon cancers, and one breast cancer). The lesions were confirmed to be malignant by means of biopsy, surgery, or a minimum clinical follow-up of six months. In addition, seven regions showing slight accumulation of FDG (standardized uptake value $<2.5$ ) were selected as reference organs, and their ADCs (in $\times 10^{-3} \mathrm{~mm}^{2} / \mathrm{s}$ ) were measured and compared with those of the malignant lesions. Twentyfive (92.6\%) of 27 malignant lesions were detected visually with DWIBS, in contrast to 22 malignant lesions (81.5\%) with FDG-PET/CT. However, there was no significant difference between the mean ADCs of the reference organs $(n=7,1.54 \pm 0.24 \mathrm{SD})$ and the malignant lesions $(n=25$, $1.18 \pm 0.70 \mathrm{SD})$. Unfortunately, diagnostic performance of DWIBS in terms of sensitivity and specificity was not assessed in this study. Furthermore, the heterogeneity of the patient population may have led to overlapping ADCs in reference organs and malignant lesions. Another drawback of this study is that it was not clear whether the readers were blinded to the reference standard and FDG-PET/CT when interpreting the DWIBS images [38].

Tamai et al. [39] performed DWIBS in 18 consecutive females with surgically proven endometrial cancer and 12 
cervical cancer patients with pathologically confirmed normal endometrium. All endometrial cancers and the normal endometria appeared hyperintense on DWIBS images. The mean ADC (in $\times 10^{-3} \mathrm{~mm}^{2} / \mathrm{s}$ ) of endometrial cancer $(0.88 \pm 0.16 \mathrm{SD})$ was significantly lower $(P<0.01)$ than that of normal endometrium $(1.53 \pm 0.10 \mathrm{SD})$, without any overlap between them. The mean ADC for each histologic grade was $0.93 \pm 0.16 \mathrm{SD}$ (grade I), $0.92 \pm 0.13$ SD (grade 2), and $0.73 \pm 0.09 \mathrm{SD}$ (grade 3). The ADC of grade 3 tumors was significantly lower than that of grade 1 tumors $(P<0.05)$. This study proved DWIBS to be feasible in demonstrating endometrial cancer and suggests that ADC measurement has a potential ability to differentiate between normal and cancerous tissue in the endometrium. Estimation of histologic grade based on ADCs seems difficult because of considerable overlap. A limitation of this study is that no benign endometrial pathologies were included. Therefore, it is still unclear whether it is possible to differentiate malignant from non-malignant endometrial lesions using ADC measurements in DWIBS. ADCs of the normal endometrium may vary according to menstrual cycle in premenopausal women, but menstrual phase of the patients was not taken into consideration in analyzing the ADCs of the normal endometrium in the control group. Another drawback of this study was the fact that readers were not completely blinded when analyzing the images of the control group [39].

Tamai et al. [40] also performed DWIBS in 43 surgically treated patients with 58 myometrial tumors, including seven uterine sarcomas (five leiomyosarcomas and two endometrial stromal sarcomas) and 51 benign leiomyomas (43 ordinary leiomyomas, two cellular leiomyomas and six degenerated leiomyomas), in addition to conventional nonenhanced and postcontrast MRI sequences. Both uterine sarcomas and cellular leiomyomas exhibited high signal intensity on DWIBS images, whereas ordinary leiomyomas and most degenerated leiomyomas showed low signal intensity. The mean ADC (in $\times 10^{-3} \mathrm{~mm}^{2} / \mathrm{s}$ ) of sarcomas $(1.17 \pm 0.15 \mathrm{SD})$ was lower than those of the normal myometrium ( $1.62 \pm 0.11 \mathrm{SD})$ and degenerated leiomyomas $(1.70 \pm 0.11 \mathrm{SD})$ without any overlap; however, they overlapped with those of ordinary leiomyomas and cellular leiomyomas. This study suggests that, in addition to morphological features, DWIBS may be able to distinguish uterine sarcomas from benign leiomyomas, although ADC measurement may have a limited role due to a large overlap between sarcomas and benign leiomyomas. A disadvantage of this study is the nonconsecutive enrollment of patients. Furthermore, menstrual phase of the patients was not taken into account in the assessment of the ADC of the normal myometrium [40].

Ballon et al. [17] investigated the feasibility of monitoring response to therapy using DWIBS. Examples presented in their study include the evaluation of therapeutic response in bone marrow during cytotoxic therapy for leukemia and metastatic prostate cancer, and during cytokine administration for marrow mobilization prior to stem cell harvest [17].

\section{Drawbacks and limitations of DWIBS}

\section{General}

Although DWIBS offers many potential applications in oncological imaging, it may suffer from several drawbacks. First, DWIBS does not exclusively visualize malignant tumors. Benign pathologies with restricted diffusion, such as abscesses, will also exhibit high signal on DWIBS source images (Fig. 11) [41]. Furthermore, DWIBS not only visualizes pathological areas of restricted diffusion, but also several normal structures; brain, salivary glands, tonsils, spleen, gallbladder, small intestine/small intestinal contents, colon, adrenal glands, prostate, testes, penis, endometrium, ovaries, spinal cord, peripheral nerves, lymph nodes, and bone marrow may all exhibit high signal intensity on DWIBS source images (Figs. 4, 5, 10). High signal intensities within these organs do not necessarily indicate pathologic states, whereas true lesions within these organs may be obscured. Visibility of the spleen is highly variable and probably dependent on its iron content (and consequent signal loss). T2 shine-through and insufficiently suppressed fat signal may also hinder image interpretation. Although at the expense of prolonged acquisition time, $\mathrm{T} 2$ shine-through effects can be removed by performing $\mathrm{ADC}$ mapping, provided that $\mathrm{ADC}$ mapping is accurate and feasible in DWIBS. Another limitation of DWIBS is its suppression of background body signals, as a result of which sufficient anatomical information is lacking. Standard T1- and T2-weighted sequences therefore remain indispensable to act as an anatomical reference frame for the DWIBS images, in order to exactly localize lesions. DWIBS and anatomical (whole-body) MRI can be viewed side-by side, or fused with commercially available software (Fig. 12). An additional advantage of DWIBS/ anatomical MRI fusion may be increased sensitivity and specificity [37]. Finally, although DWIBS images can be post-processed to create MIPs and PET-like images, which are very attractive for demonstration purposes (Figs. 4, 5, 10), source images should always be consulted, because subtle lesions may be missed or obscured in projected images.

\section{ADC measurements in DWIBS}

Signal intensity is directly related to the degree of diffusion and the $\mathrm{ADC}$ is the quantitative measure of diffusion in DWI. Both signal intensity and ADCs are mainstays in the identification and characterization of lesions in DWI. However, because of the allowance of respiratory motion in DWIBS, slice levels of images obtained with different $b$ - 


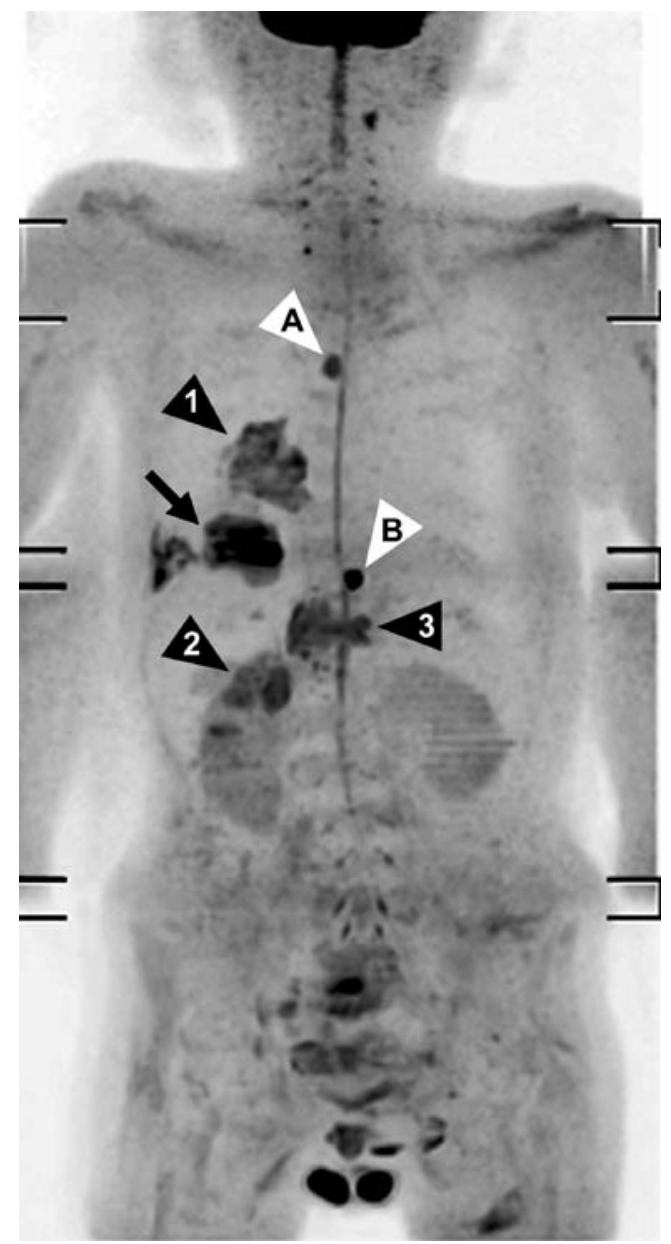

Fig. 11 A 78-year-old man with metastatic lung cancer and two benign lesions visualized with DWIBS. Coronal maximum intensity projection DWIBS image shows an area with restricted diffusion in the right lower lobe, consistent with lung cancer (arrow). In addition, DWIBS image shows ipsilateral hilar lymph node metastasis (arrowhead 1), retroperitoneal/adrenal metastasis (arrowhead 2), and vertebral metastasis (arrowhead 3). DWIBS image also shows restricted diffusion in a thoracic vertebra (arrowhead A). T1and T2-weighted images (not shown), however, indicate this "lesion" to be a (benign) cavernous hemangioma, instead of a vertebral metastasis. Furthermore, DWIBS image shows a wellcircumscribed area of restricted diffusion in the epigastric region (arrowhead B); physical examination reveals a (benign) sebaceous cyst to be responsible for this area of restricted diffusion

values may not be identical. In addition, because DWIBS employs multiple slice excitations, slices levels of images obtained with the same $b$-value may be different. Consequently, ADC measurements of moving organs in DWIBS may be less accurate, less reproducible, and different from conventional (breathhold or respiratory triggered) DWI. In other words, DWIBS images of moving organs are possibly more suitable for visual nonquantitative evaluation than quantitative analysis. In their phantom study, Muro et al. [42] reported that ADCs of slowly moving phantoms, simulating respiratory motion, were no more than $10 \%$ different from ADCs of static phantoms, if the ROIs were placed (and remained) within the homogeneous phantom. However, if the ROIs covered the edge of the phantom, ADCs were markedly different [42]. Thus, the results of this study indicate that ADC measurements of homogeneous tissue appear to be accurate, whereas ADC measurements of heterogeneous tissue or small lesions (e.g., pulmonary nodules or small liver lesions) may be unreliable in DWIBS. Nasu et al. [43] compared ADCs of hepatic malignant tumors in both respiratory triggered DWI and DWIBS. Thirty patients with liver metastasis or hepatocellular carcinoma in the right hepatic lobe were examined. The mean measured $\mathrm{ADC}$ in respiratory triggered DWI was $1.36( \pm 0.33 \mathrm{SD})$, whereas the mean measured ADC in DWIBS was $1.47( \pm 0.61 \mathrm{SD})$. The statistical difference between both mean ADCs was not clear $(P=0.050)$. The individual ADCs in DWIBS were more scattered than those in respiratory triggered DWI and there was no regular pattern in individual ADC differences [43]. It should be noted, however, that the comparison between respiratory triggered DWI and DWIBS was (clinically) not fair, since roughly estimated scanning time of respiratory triggered DWI was approximately 1.3- to 2-times longer [43]. In a similar investigation with accurate time measurements, scanning time in respiratory triggering was even nearly three-times longer [44]. More in-vivo research is needed to determine the accuracy and reproducibility of ADC measurements in DWIBS.

\section{Future considerations of DWIBS}

More studies with large sample sizes are needed to establish the value of DWIBS and to develop criteria for differentiation between malignant and non-malignant lesions. Future research is also warranted to optimize the sequence for DWIBS. An important issue in DWIBS is the optimization of background body signal suppression, while maintaining high signal from areas with restricted diffusion. Fat suppression is essential for lymph node and lesion conspicuity in DWIBS. Takahara et al. [16] reported STIR to be superior to CHESS for fat suppression in the neck/ upper chest region in DWIBS at $1.5 \mathrm{~T}$. In addition, STIR has the potential advantage to avoid false positive results because of intestinal contents. However, there is no report yet as to which technique is better for detecting intestinal (e.g., colonic) tumors. Whether STIR or CHESS should be preferred for DWIBS of the trunk at $1.5 \mathrm{~T}$ requires further systematic investigation. Additionally, the utility of using negative oral contrast agents should be investigated; the use of a negative oral contrast agent may further aid in the suppression of bowel signal [45], which is especially important when evaluating (possible) gastrointestinal/intra- 

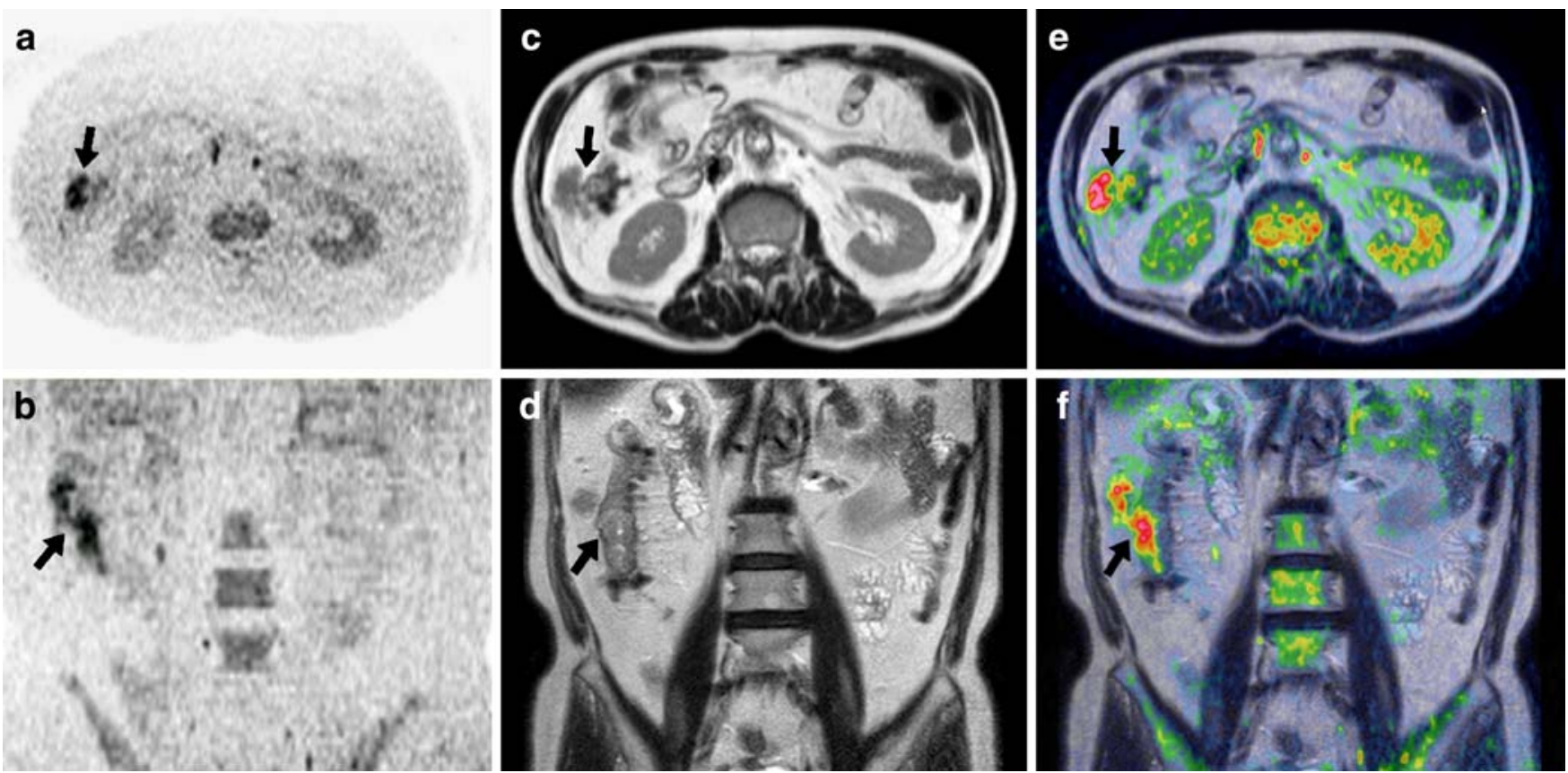

Fig. 12 Fusion of a DWIBS image and a T2-weighted image in a 64-year-old man with ascending colon cancer. a Axial and b coronally reformatted DWIBS images show abnormal signal in the area of the ascending colon. c Axial and d coronal T2-weighted

images show a large mass of intermediate signal intensity in the ascending colon, extending beyond the serosa. e Fused axial and $\mathbf{f}$ coronal DWIBS/T2-weighted images, highlighting the lesion

abdominal malignancies. DWIBS at $3.0 \mathrm{~T}$ potentially offers higher SNR, since SNR increases linearly with increasing field strength. Susceptibility artifacts, on the other hand, increase exponentially with increasing field strength, which will degrade DWIBS images [46]. A recent feasibility study indeed reported DWIBS at $3.0 \mathrm{~T}$ to provide a better lesion-to-bone tissue contrast, compared with DWIBS at $1.5 \mathrm{~T}$ [47]. STIR proved to offer the best fat suppression in all body regions at 3.0 T [47]. However, larger susceptibility-induced image distortions and signal intensity losses, stronger blurring artifacts, and more pronounced motion artifacts degraded the image quality at $3.0 \mathrm{~T}$ [47]. Thus, further investigations concerning DWIBS at 3.0 T should be undertaken. Image acquisition

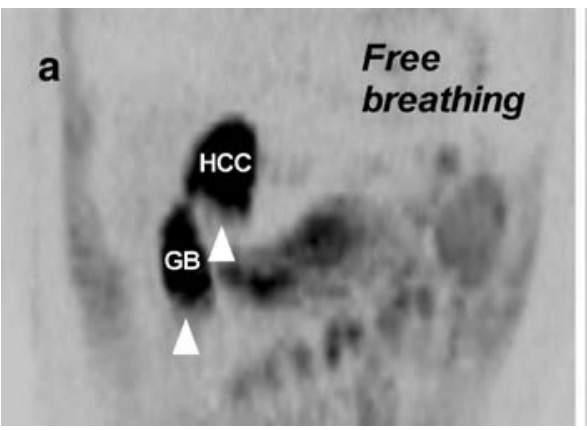

Fig. 13 Comparison of DWIBS images obtained (1) during free breathing, (2) with respiratory triggering, and (3) with TRON, in a 48-year-old male with hepatocellular carcinoma and hemochromatosis. a Coronally reformatted DWIBS image obtained during free breathing shows an area with restricted diffusion in segment IV of the liver; biopsy proved this lesion to be a hepatocellular carcinoma $(H C C)$. Bile in the gallbladder $(G B)$ also shows restricted diffusion. Note significant blurring of the hepatocellular carcinoma and the gallbladder, especially at their lower boundaries (arrowheads). b Coronally reformatted DWIBS image obtained with respiratory triggering results in reduced blurring of the hepatocellular carcinoma $(H C C)$ and the gallbladder $(G B)$, but introduces stepladder-like artifacts due to gating error. Furthermore, image acquisition time is approximately 2.5 -times prolonged in comparison with a. c Coronally reformatted DWIBS image obtained with TRON shows slight gating error, but offers good delineation of the hepatocellular carcinoma $(H C C)$ and the gallbladder $(G B)$. Image acquisition time is only slightly (approximately 1.2-times) increased in comparison with a 
during free breathing is the hallmark and the driving force behind the DWIBS sequence. A disadvantage of this free breathing approach, however, is the possibility of image blurring due to the periodic displacement of the diaphragm (Fig. 13a), which may hamper adequate visualization of small lesions near the diaphragm, such as in the liver. Respiratory triggering may be used to reduce image blurring (Fig. 13b), but increases image acquisition time. The recently introduced tracking only navigator (TRON) technique, however, adequately deals with both problems. As the name implies, the navigator-echo in TRON is not used to create a gating window (in other words, data acquired during the entire breathing cycle can be used for image formation), but is only used to track and correct for displacements. Consequently, displaced organs and lesions near the diaphragm can be visualized with less blurring and with only minimal prolongation of scan time (Fig. 13c) [44]. TRON is a very promising tool for DWIBS of paradiaphragmatic organs and lesions, but its technical performance (i.e. whether it routinely results in anatomically faithful, reproducible images) still needs to be investigated.

\section{Summary}

Image acquisition during free breathing, multiple signal averaging, and background body signal suppression by means of a fat suppression pre-pulse and heavy diffusion weighting, are the main features of DWIBS. DWIBS is a diffusion-weighted sequence, but its (image) characteristics are different from conventional (breathhold or respiratory triggered) DWI. DWIBS highlights areas with restricted diffusion, such as occurs in many malignant primary and metastatic tumors, and provides an outstanding visualization of lymph nodes. Although the exact value of DWIBS still has to be established, it has potential use in tumor staging, monitoring response to cancer therapy, and in the detection of tumor persistence or recurrence.

Open Access This article is distributed under the terms of the Creative Commons Attribution Noncommercial License which permits any noncommercial use, distribution, and reproduction in any medium, provided the original author(s) and source are credited.

\section{References}

1. World Health Organization (2005) International union against cancer. Global action against cancer. WHO Press, Geneva

2. Ichikawa T, Haradome H, Hachiya J, Nitatori T, Araki T (1999) Diffusionweighted MR imaging with single-shot echo-planar imaging in the upper abdomen: preliminary clinical experience in 61 patients. Abdom Imaging 24:456-461

3. Ichikawa T, Haradome H, Hachiya J, Nitatori T, Araki T (1998) Diffusionweighted MR imaging with a singleshot echoplanar sequence: detection and characterization of focal hepatic lesions. AJR Am J Roentgenol 170:397-402

4. Muller MF, Prasad P, Siewert B et al (1994) Abdominal diffusion mapping with use of a whole-body echo-planar system. Radiology 190:475-478

5. Muller MF, Prasad P, Bimmler D et al (1994) Functional imaging of the kidney by means of measurement of the apparent diffusion coefficient. Radiology 193:711-715

6. Edelman RR, Gaa J, Wedeen VJ et al (1994) In vivo measurement of water diffusion in the human heart. Magn Reson Med 32:423-428
7. Bammer R (2003) Basic principles of diffusion-weighted imaging. Eur J Radiol 45:169-184

8. Glockner JF, Hu HH, Stanley DW, Angelos L, King K (2005) Parallel MR imaging: a user's guide. Radiographics 25:1279-1297

9. Thoeny HC, De Keyzer F (2007) Extracranial applications of diffusionweighted magnetic resonance imaging. Eur Radiol 17:1385-1393

10. Koh DM, Collins DJ (2007) Diffusionweighted MRI in the body: applications and challenges in oncology. AJR Am J Roentgenol 188:1622-1635

11. Low RN, Gurney J (2007) Diffusionweighted MRI (DWI) in the oncology patient: value of breathhold DWI compared to unenhanced and gadolinium-enhanced MRI. J Magn Reson Imaging 25:848-858

12. Nasu K, Kuroki Y, Nawano S et al (2006) Hepatic metastases: diffusionweighted sensitivity-encoding versus SPIO-enhanced MR imaging. Radiology 239:122-130

13. Yoshikawa T, Kawamitsu H, Mitchell DG et al (2006) ADC measurement of abdominal organs and lesions using parallel imaging technique. AJR Am J Roentgenol 187:1521-1530

14. Nasu K, Kuroki Y, Kuroki S, Murakami K, Nawano S, Moriyama N (2004) Diffusion-weighted single shot echo planar imaging of colorectal cancer using a sensitivity-encoding technique. Jpn J Clin Oncol 34:620-626
15. Taouli B, Martin AJ, Qayyum A et al (2004) Parallel imaging and diffusion tensor imaging for diffusion-weighted MRI of the liver: preliminary experience in healthy volunteers. AJR Am J Roentgenol 183:677-680

16. Takahara T, Imai Y, Yamashita T, Yasuda S, Nasu S, Van Cauteren M (2004) Diffusion weighted whole body imaging with background body signal suppression (DWIBS): technical improvement using free breathing, STIR and high resolution 3D display. Radiat Med 22:275-282

17. Ballon D, Watts R, Dyke JP et al (2004) Imaging therapeutic response in human bone marrow using rapid whole-body MRI. Magn Reson Med 52:1234-1238

18. Stejskal EO, Tanner JE (1965) Spin diffusion measurements: spin echoes in the presence of a time-dependent field gradient. J Chem Phys 42:288-292

19. Turner R, Le Bihan D, Maier J et al (1990) Echo-planar imaging of intravoxel incoherent motion. Radiology 177:407-414

20. Le Bihan D, Breton E, Lallemand D, Aubin ML, Vignaud J, Laval-Jeantet M (1988) Separation of diffusion and perfusion in intravoxel incoherent motion MR imaging. Radiology 168:497-505 
21. Le Bihan D, Breton E, Lallemand D, Grenier P, Cabanis E, Laval-Jeantet M (1986) MR imaging of intravoxel incoherent motions: application to diffusion and perfusion in neurological disorders. Radiology 161:401-407

22. Le Bihan D (1991) Molecular diffusion nuclear magnetic resonance imaging. Magn Reson Q 7:1-30

23. Provenzale JM, Mukundan S, Barboriak DP (2006) Diffusionweighted and perfusion MR imaging for brain tumor characterization and assessment of treatment response. Radiology 239:632-649

24. Delfaut EM, Beltran J, Johnson G Rousseau J, Marchandise X, Cotten A (1999) Fat suppression in MR imaging: techniques and pitfalls. Radiographics 19:373-382

25. Li S, Sun F, Jin ZY, Xue HD, Li ML (2007) Whole-body diffusion-weighted imaging: technical improvement and preliminary results. J Magn Reson Imaging 26:1139-1144

26. Torabi M, Aquino SL, Harisinghani MG (2004) Current concepts in lymph node imaging. J Nucl Med 45:15091518

27. Will O, Purkayastha S, Chan $\mathrm{C}$ et al (2006) Diagnostic precision of nanoparticle-enhanced MRI for lymphnode metastases: a meta-analysis. Lancet Oncol 7:52-60

28. Jaffe CC (2006) Measures of response: RECIST, WHO, and new alternatives. J Clin Oncol 24:3245-3251

29. Weber WA (2006) Positron emission tomography as an imaging biomarker. J Clin Oncol 24:3282-3292

30. Hamstra DA, Rehemtulla A, Ross BD (2007) Diffusion magnetic resonance imaging: a biomarker for treatment response in oncology. J Clin Oncol 25:4104-4109
31. Hamstra DA, Chenevert TL, Moffat BA et al (2005) Evaluation of the functional diffusion map as an early biomarker of time-to-progression and overall survival in high-grade glioma Proc Natl Acad Sci USA 102:1675916764

32. Moffat BA, Chenevert TL, Lawrence TS et al (2005) Functional diffusion map: a noninvasive MRI biomarker for early stratification of clinical brain tumor response. Proc Natl Acad Sci USA 102:5524-5529

33. Lee KC, Bradley DA, Hussain M et al (2007) A feasibility study evaluating the functional diffusion map as a predictive imaging biomarker for detection of treatment response in a patient with metastatic prostate cancer to the bone. Neoplasia 9:10031011

34. Rohren EM, Turkington TG, Coleman RE (2004) Clinical applications of PET in oncology. Radiology 231:305-332

35. Ichikawa T, Erturk SM, Motosugi U et al (2006) High-B-value diffusionweighted MRI in colorectal cancer. AJR Am J Roentgenol 187:181184

36. Ichikawa T, Erturk SM, Motosugi U et al (2007) High-b value diffusionweighted MRI for detecting pancreatic adenocarcinoma: preliminary results. AJR Am J Roentgenol 188:409-414

37. Tsushima Y, Takano A, TaketomiTakahashi A, Endo K (2007) Body diffusion-weighted MR imaging using high b-value for malignant tumor screening: usefulness and necessity of referring to $\mathrm{T} 2$-weighted images and creating fusion images. Acad Radiol 14:643-650

38. Komori T, Narabayashi I, Matsumura K et al (2007) 2-[Fluorine-18]-fluoro-2deoxy-D-glucose positron emission tomography/computed tomography versus whole-body diffusion-weighted MRI for detection of malignant lesions: initial experience. Ann Nucl Med 21:209-215
39. Tamai K, Koyama T, Saga $T$ et al (2007) Diffusion-weighted MR imaging of uterine endometrial cancer. $\mathrm{J}$ Magn Reson Imaging 26:682-687

40. Tamai K, Koyama T, Saga T et al (2007) The utility of diffusionweighted MR imaging for differentiating uterine sarcomas from benign leiomyomas. Eur Radiol DOI 10.1007/ s00330-007-0787-7

41. Stadnik TW, Demaerel P, Luypaert RR et al (2003) Imaging tutorial: differential diagnosis of bright lesions on diffusion-weighted MR images. Radiographics 23:e7

42. Muro I, Takahara T, Horie $\mathrm{T}$ et al (2005) Influence of respiratory motion in body diffusion weighted imaging under free breathing (examination of a moving phantom). Nippon Hoshasen Gijutsu Gakkai Zasshi 61:1551-1558

43. Nasu K, Kuroki Y, Sekiguchi R, Nawano S (2006) The effect of simultaneous use of respiratory triggering in diffusion-weighted imaging of the liver. Magn Reson Med Sci 5:129-136

44. Takahara T, Ogino T, Okuaki T et al (2007) Respiratory gated body diffusion weighted imaging avoiding prolongation of scan time: tracking only navigator echo (TRON) technique. Presented at the Joint Annual Meeting ISMRM-ESMRMB, Berlin, Germany, May 19-25, 2007

45. Laghi A, Paolantonio P, Iafrate F, Altomari F, Miglio C, Passariello R (2002) Oral contrast agents for magnetic resonance imaging of the bowel. Top Magn Reson Imaging 13:389-396

46. Kuhl CK, Textor J, Gieseke J et al (2005) Acute and subacute ischemic stroke at high-field-strength (3.0-T) diffusion-weighted MR imaging: intraindividual comparative study. Radiology 234:509-516

47. Murtz P, Krautmacher C, Traber F, Gieseke J, Schield HH, Willinek WA (2007) Diffusion-weighted whole-body MR imaging with background body signal suppression: a feasibility study at 3.0 Tesla. Eur Radiol 17:3031-3037 